\title{
Interrupted aortic arch in an adult
}

\author{
T Kumanan ${ }^{1}$, M Guruparan², K Gnanakanthan ${ }^{3}$, U K Ratnayake ${ }^{1}$ \\ Ceylon Medical Journal 2016; 61: 135-136 ～DOI: http://doi.org/10.4038/cmj.v61i3.8353
}

\section{Introduction}

Interrupted Aortic Arch (IAA) is an uncommon congenital anomaly representing approximately $1-2 \%$ of congenital heart disease. More than $97 \%$ of the cases have associated cardiac abnormalities, mainly VSD. The median age at death in untreated cases is four days making it rare to survive into adulthood without an associated structural cardiac abnormality. Here we report a patient with an IAA without any structural cardiac lesion who survived into third decade of his life.

\section{Case report}

A 25-year old man presented with acute onset of severe headache, weakness and numbness of right hand. On examination the radial pulses were synchronous and normal in volume but the lower limb pulses were faint. A radial-femoral delay was noted. His blood pressure was 192/108 mmHg.

CT scan of brain revealed a small thalamic haemorrhage compatible with his neurological deficit. The 2D Echocardiogram confirmed marked left ventricular hypertrophy and ruled out congenital cardiac defects. Notching of the fifth to eighth ribs posteriorly was noted on chest X-ray. A contrast enhanced CT angiography revealed a diagnosis of interrupted aortic arch (IAA - type A). He was referred to cardiothoracic team and underwent surgical intervention.

The operation was done through the standard median sternotomy. The ascending aorta was completely mobilised up to the aortic arch. The arterial cannulation was performed in the aortic arch below the innominate artery. Following right atrial cannulation cardiopulmonary bypass was established with systemic cooling of $32^{\circ} \mathrm{C}$. Following cardiac arrest the apex of the heart was then elevated out of the pericardial wall to expose the posterior pericardium. The descending aorta and oesophagus were palpated. The pericardium was opened in a longitudinal fashion from the diaphragmatic recess to just inferior to the pericardial reflection onto the left inferior pulmonary veins. The partial occlusion clamp was then applied to the anterior wall of the exposed descending thoracic aorta. The longitudinal aortotomy was made and end to side anastomosis was constructed using 4/0 polypropylene running suture (Figures 1 and 2). After routing the graft behind the IVC, a clamp was placed onto the mid portion of the graft.The proximal graft anastomosis was performed with the heart arrested.

This procedure is being done for the interrupted aortic arch and coarctation with associated intracardiac anaomaly which requires surgical correction at the same time as one off procedure. Since this patient had extensive collaterals on the chest wall and the coarctated segment was long resection and end to end anastomosis was not possible and would have led to excessive bleeding from the collaterals if surgery was done via thoracotomy. The patient had an uneventful recovery and was maintained on minimal antihypertensive medication and is leading a normal healthy life.

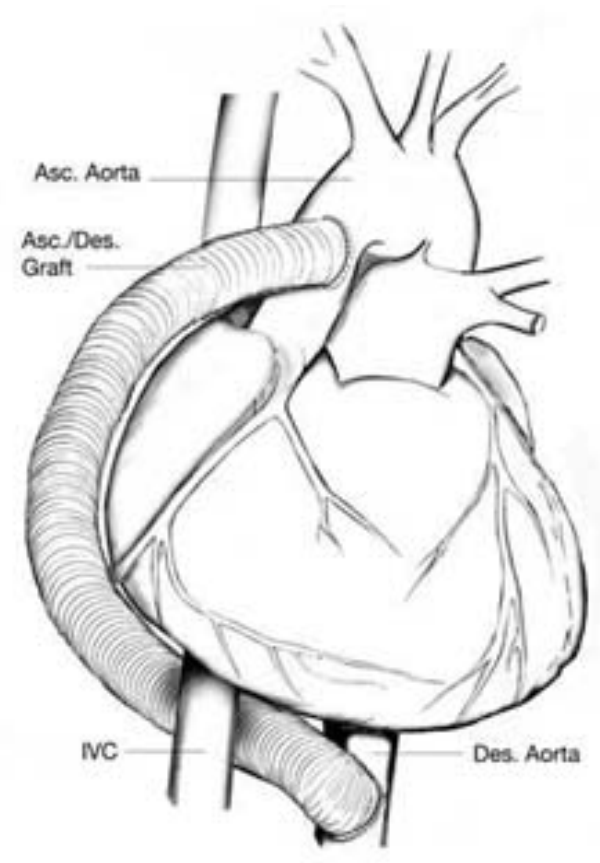

Figure 1.

${ }^{1}$ University Medical Unit, ${ }^{2}$ Teaching Hospital Jaffna and ${ }^{3}$ Teaching Hospital Kandy, Sri Lanka.

Correspondence: TK, e-mail: <mtkumanan@yahoo.com>. Received 26 January and revised version accepted 31 May 2016.

This is an open-access article distributed under the terms of the Creative Commons Attribution License, which permits unrestricted use, distribution, and reproduction in any medium, provided the original author and source are credited. 


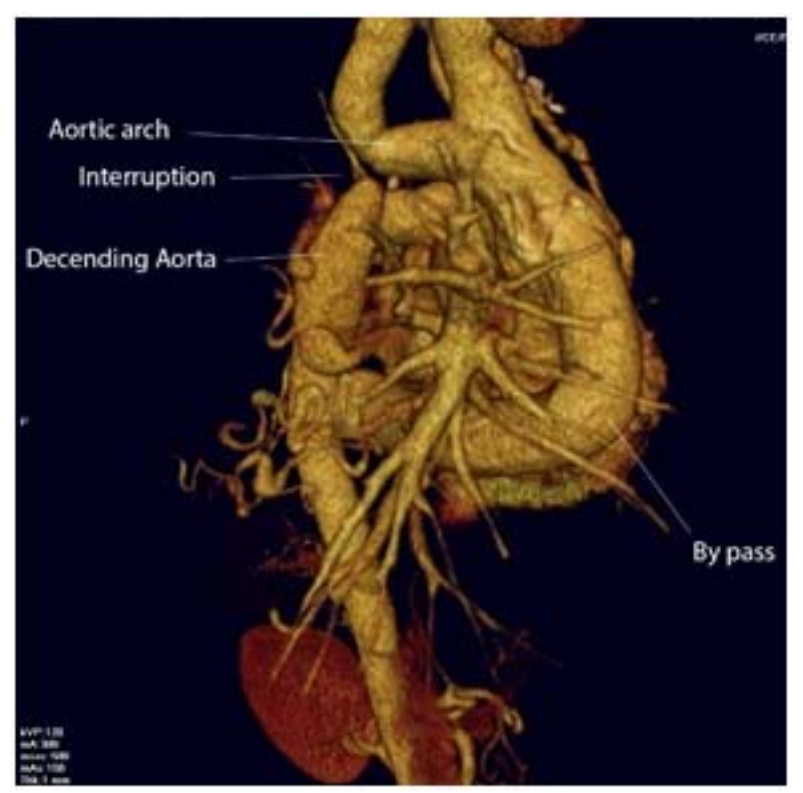

Figure 2.

\section{Discussion}

IAA is a rare congenital malformation that occurs in 3 per million live births. Defined as loss of luminal continuity between the ascending and descending portions of the aorta, it is associated with very poor prognosis without surgical intervention [1]. IAA is subdivided into types $\mathrm{A}, \mathrm{B}$ and $\mathrm{C}$ according to the anatomical site of the interruption [2].

Type A: The interruption occurs just beyond the left subclavian artery. Approximately 30 to 40 percent of the infants with interrupted aortic arch have type A.

Type B: The interruption occurs between the left carotid artery and the left subclavian artery. It is the most common form of interrupted aortic arch. It accounts for about 53 percent of reported cases.
Type C: The interruption occurs between the innominate artery and the left carotid artery. It is the least common form of interrupted aortic arch, accounting for about 4 percent of reported cases.

IAA is usually diagnosed soon after birth, and if left untreated, $90 \%$ of the affected infants die at a median age of 4 days. IAA is almost always associated with a large ventricular septal defect (VSD) [3]. Absence of VSD and a presenting during the third decade of life has not been described before. Definitive management of IAA in adults is surgical, either as direct anastomosis or conduit interposition [4].

\section{Consent}

Patient gave informed written consent for this publication.

\section{Conflicts of interest}

There are no conflicts of interest.

\section{References}

1. Messner G, Reul GJ, Flamm SD, et al. Interrupted Aortic Arch in an Adult: Single-Stage Extra-Anatomic Repair. Tex Heart Inst J 2002; 29: 118-21.

2. Celoria GC, Patton RB. Congenital absence of aortic arch. Am Heart J 1959; 58: 407-13.

3. Jacobs ML, Chin AJ, Rychik J. et al. Interrupted Aortic Arch: Impact of Subaortic Stenosis on Management and Outcome. Circulation 1995; 92: 128-31.

4. Reardon MJ, Hallman GL, Cooley DA. Interrupted Aortic Arch: Brief Review and Summary of an Eighteen-Year Experience. Tex Heart Inst J 1984; 11: 250-9. 